\section{Damaged good - targeting DNA damage repair in neuroendocrine disease}

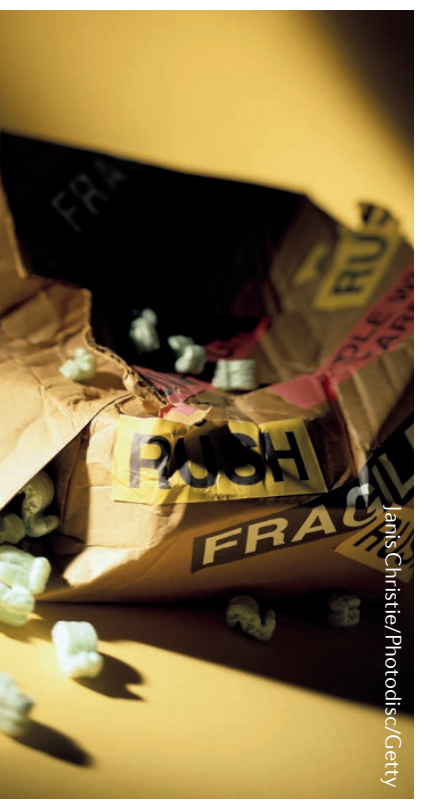

Conversion to a neuroendocrine phenotype in prostate cancer is associated with overexpression of $M Y C N$ and its protein product N-MYC. N-MYC has been shown to drive deveopment of neuroendocrine prostate cancer (NEPC) from prostate epithelial cells and is linked to the expression of DNA damage repair (DDR) genes. Around 25\% of metastatic castration-resistant prostate cancer (CRPC) harbours genomic changes in DDR genes, which are associated with improved responses to olaparib, a poly(ADP-ribose) polymerase (PARP) inhibitor.

Zhang and colleagues used a bioinformatics approach to sequence data from publicly available data sets and patient-derived xenograft (PDX) models to identify MYCN-regulated
PARP-DDR pathways in NEPC development that could be targeted for therapy. Comparison of PDX models and public databases showed similar DDR-mitotic (DDR-M) gene expression patterns with upregulation of $\sim 75 \%$ of DDR-M genes, downregulation of $\sim 25 \%$ of DDR-M genes and a correlation between upregulation of DDR-M gene expression and neuroendocrine CRPC development. Clustering analysis showed that the upregulation of the majority of these genes is positively correlated with loss of or reduced $A R$ expression and increased MYCN expression. Genes in the N-MYC and PARP1/2-DDR signalling pathway were uniformly increased in NEPC compared with adenocarcinoma. DDR-M genes that are involved in
MYCN-PARP1/2-DDR signalling were further investigated using ChIP analysis, which confirmed that N-MYC directly binds the promoter regions of $P A R P 1$, PARP2, BRCA1, RMI2, and TOPBP1. Knockdown of MYCN or PARP1 reduced expression of MYCN-PARP1/2-DDR pathway genes and neuroendocrine markers, and inhibition with small interfering RNA (siRNA) suppressed oncogenicity.

Finally, the authors treated cell lines and PDX models with olaparib and an AURKA inhibitor, and showed similar effects to those seen with siRNA.

Targeting of this novel DNA damage repair pathway in CRPC could provide a new therapeutic strategy for neuroendocrine disease.

Annette Fenner

ORIGINAL ARTICLE Zhang, W. et al. Targeting the MYCN-PARP-DNA damage response pathway in neuroendocrine prostate cancer. Clin. Cancer Res. http://dx.doi.org/10.1158/1078-0432.CCR17-1872 (2017) 